\title{
Continuous Tensor Network States for Quantum Fields
}

\author{
Antoine Tilloy ${ }^{*}$ and J. Ignacio Cirac ${ }^{\dagger}$ \\ Max-Planck-Institut für Quantenoptik, Hans-Kopfermann-Straße 1, 85748 Garching, Germany
}

(Received 3 August 2018; revised manuscript received 12 February 2019; published 28 May 2019)

\begin{abstract}
We introduce a new class of states for bosonic quantum fields which extend tensor network states to the continuum and generalize continuous matrix product states to spatial dimensions $d \geq 2$. By construction, they are Euclidean invariant and are genuine continuum limits of discrete tensor network states. Admitting both a functional integral and an operator representation, they share the important properties of their discrete counterparts: expressiveness, invariance under gauge transformations, simple rescaling flow, and compact expressions for the $N$-point functions of local observables. While we discuss mostly the continuous tensor network states extending projected entangled-pair states, we propose a generalization bearing similarities with the continuum multiscale entanglement renormalization ansatz.
\end{abstract}

DOI: 10.1103/PhysRevX.9.021040

Subject Areas: Particles and Fields, Quantum Physics, Strongly Correlated Materials

\section{INTRODUCTION}

Tensor network states (TNSs) provide an efficient parametrization of physically relevant many-body wave functions on the lattice $[1,2]$. Obtained from a contraction of low-rank tensors on so-called virtual indices, they economically approximate the states of systems with local interactions in thermal equilibrium. Their number of parameters scales only polynomially with the lattice size $[3,4]$, circumventing the exponential growth of the Hilbert space dimension. TNSs have led to powerful numerical methods to compute the physical properties of complex system [5-7], most notably in one spatial dimension $d=1$, where matrix product states (MPSs) [8], the simplest incarnation of TNSs, are at the basis of what is arguably the most successful method to describe strongly correlated systems [9-11]. In higher dimensions $d \geq 2$, accurate results [12] have also been obtained using projected entangled-pair states (PEPSs) [13], a natural generalization of MPSs. Another family of TNSs, the multiscale renormalization ansatz (MERA) [14], has proved well suited to describe scale-invariant states $[15,16]$ appearing in critical phenomena.

Beyond numerical computations, TNSs provide important insights into the nature of many-body quantum systems

\footnotetext{
*antoine.tilloy@mpq.mpg.de

ignacio.cirac@mpq.mpg.de
}

Published by the American Physical Society under the terms of the Creative Commons Attribution 4.0 International license. Further distribution of this work must maintain attribution to the author(s) and the published article's title, journal citation, and DOI. and have helped describe and classify their physical properties. By design, their entanglement obeys the area law [17-19], which is a fundamental property of low-energy states of systems with local interactions. They enable a succinct classification of symmetry-protected [20-23] and topological phases of matter [24,25]. TNSs also have a builtin bulk-boundary correspondence [26], which makes close connections to physical phenomena appearing in exotic materials $[27,28]$. Finally, they can be used to build toy models illustrating the holographic principle and the celebrated AdS/CFT correspondence [29-31].

For regular spin lattices, PEPSs assign a tensor to each lattice site, with $2 z$ virtual and one physical (spin) indices, where $z$ is the coordination number. The virtual indices are contracted according to the lattice geometry, yielding a wave function for the spin degrees of freedom. This description is particularly useful in translationally invariant systems, as this symmetry may simply be imposed by choosing the same tensor on each site. For MERA [14,32], a treelike structure of two types of tensors is used. In both cases, the whole many-body wave function is determined by one or a few tensors, which encode all the physical properties.

An important challenge in the theory of TNS is the generalization from lattice to continuous systems. Such an extension would allow the direct study of quantum field theories, without the need for a prior breaking of spatial symmetries with a discretization. Furthermore, the continuum provides a whole range of exact and approximate analytic techniques (such as exact Gaussian functional integrals, saddle-point approximations, or diagrammatic expansions) that have no obvious discrete counterparts and that could provide useful additions to the TNS toolbox. 
A natural way to carry out such a program is to simply take the continuum limit of a TNS, by letting the lattice spacing tend to zero while appropriately rescaling the tensors. In fact, this task has been carried in one spatial dimension, $d=1$, where it yields continuous matrix product states (CMPSs) $[33,34]$. In higher dimensions, however, the situation does not seem trivial. Naive extensions of CMPSs have a preferred spatial direction and break Euclidean symmetries [35]. In Ref. [35], a proposal for CPEPS was put forward to overcome such a limitation, but the resulting state was no longer obtained from the continuum limit of a TNS. Thus, so far there seems to be no fully satisfactory way of extending TNSs to the continuum in $d \geq 2$.

In this article, we propose a definition of continuous tensor network states (CTNSs) that naturally extends TNSs to the continuum. We obtain them as a genuine continuum limit of TNSs but manage to preserve Euclidean invariance. As in previous works [35-37], we exploit the similarity between a tensor contraction over the indices lying on the links of a tensor network and a functional integral over a field living on the continuum limit of this mesh. The key difference lies in the way the continuum limit is taken in higher dimensions: As we argue, the $d=1$ case of CMPSs is too peculiar to be directly extended.

The first definition of CTNS we propose in Sec. II takes the form of a functional integral over auxiliary scalar fields as advertised. From this definition, which makes local Euclidean invariance manifest, we derive an operator representation similar to the one used for CMPSs. Importantly, we show in Sec. III how this ansatz can be obtained from a continuum limit of a discrete TNS. We then study some of its properties reminiscent of the discrete: its ability to approximate (possibly inefficiently) all states (Sec. IVA), its redundancy under some so-called gauge transformations (Sec. IV B), which play a crucial role for PEPSs, its flow under scaling transformations (Sec. IV C), and its CMPS approximation in some carefully chosen limit (Sec. IV D). We then propose various methods to carry computations with Gaussian and non-Gaussian CTNSs (Sec. V). While most of our approach is aimed at the continuum limit of PEPSs, we finally generalize it to MERA-like states (and more exotic TNSs) in arbitrary dimensions by including a metric and restricting physical fields to a boundary (Sec. VI).

\section{CONTINUOUS TENSOR NETWORK STATES}

We start by giving two equivalent definitions of continuous tensor network states, leveraging a functional integral and an operator representation. Our objective at this stage is only to provide a definition of a class of states for bosonic quantum fields, with only a crude intuition for why such an object could indeed be a good definition of a CTNS. We forgo the derivation of this CTNS from a class of discrete tensor networks to the following section.

\section{A. Functional integral representation}

\section{State definition}

We begin with the functional integral representation. It will be the most direct to derive from the discrete and makes Euclidean symmetries manifest.

Definition 1 (functional integral formulation). - A CTNS of a bosonic quantum field on a domain $\Omega \subset \mathbb{R}^{d}$ with boundary $\partial \Omega$ is a state $|V, B, \alpha\rangle$ parametrized by two functions $V$ and $\alpha: \mathbb{R}^{D} \rightarrow \mathbb{C}$ and a boundary functional $B$ : $L^{2}(\partial \Omega) \rightarrow \mathbb{C}$ defined by the functional integral on an auxiliary $D$-component field $\phi$ :

$$
\begin{aligned}
|V, B, \alpha\rangle= & \int \mathcal{D} \phi B\left(\left.\phi\right|_{\partial \Omega}\right) \exp \left\{-\int_{\Omega} d^{d} x \frac{1}{2} \sum_{k=1}^{D}\left[\nabla \phi_{k}(x)\right]^{2}\right. \\
& \left.+V[\phi(x)]-\alpha[\phi(x)] \psi^{\dagger}(x)\right\}|0\rangle,
\end{aligned}
$$

where $|0\rangle$ is the physical Fock vacuum state, $[\psi(x)$, $\left.\psi^{\dagger}(y)\right]=\delta^{d}(x-y)$, and $\phi=\left[\phi_{k}\right]_{k=1}^{D}$. The functions $\alpha$ and $V$ may depend explicitly on the position.

The auxiliary $D$-component field $\phi$, which is integrated over in the functional integral, is the continuous equivalent of the auxiliary bond indices that are contracted in tensor network states (see Fig. 1). This intuition is made more precise in the next section. For this reason, we call $D$ the bond-field dimension. As we see in Sec. IV, the bond-field dimension $D$ bears similarities with the bond dimension $\chi$ of discrete tensor network states.

If $\Omega$ is $\mathbb{R}^{d}$ or a torus with periodic boundary conditions, $B$ can simply be set to 1 . In that case, the state and its associated properties depend only on $V$ and $\alpha$, in the same way a TNS depends only on local tensors. If $V$ and $\alpha$ do not depend explicitly on $x$, the CTNS describes a translationally invariant state. More generally, if $\partial \Omega \neq \varnothing$, the boundary functional could induce, e.g.,

(1) Dirichlet boundary conditions. $-B\left(\left.\phi\right|_{\partial \Omega}\right) \sim \delta\left(\left.\phi\right|_{\partial \Omega}\right)$ fixing $\left.\phi\right|_{\partial \Omega}=0$ in the functional integral;

(2) Neumann boundary conditions. $-B\left(\left.\phi\right|_{\partial \Omega}\right) \sim \delta(\nabla \phi \cdot \mathbf{n})$ fixing $\left.\nabla \phi \cdot \mathbf{n}\right|_{\partial \Omega}=0$, where $\mathbf{n}$ is normal to $\partial \Omega$; or

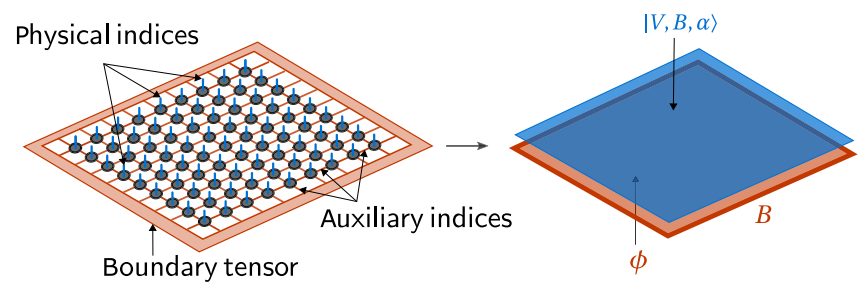

FIG. 1. Functional integral representation. In the discrete (left), a tensor network state is obtained from a contraction of auxiliary indices connecting the elementary tensors with each other and with a boundary tensor. In the continuum (right), the contraction is replaced by a functional integral (1), the auxiliary indices by fields $\phi$, and the boundary tensor by a boundary functional $B$. 
(3) something more general, given, e.g., by a quasilocal functional:

$$
B\left(\left.\phi\right|_{\partial \Omega}\right)=\exp \left\{-\oint_{\partial \Omega} d^{d-1} x \mathcal{L}[\phi(x), \nabla \phi(x)]\right\},
$$

where $\mathcal{L}$ is a function from $\mathbb{R}^{(d+1) D}$ to $\mathbb{C}$. This latter option is generated naturally when we discuss gauge invariance in Sec. IV B.

We rewrite expression (1) more explicitly as a sum over unnormalized field-coherent states. Introducing the massless free field probability measure $d \mu(\phi)$ for the auxiliary field:

$$
d \mu(\phi)=\mathcal{D} \phi \exp \left[-\frac{1}{2} \int_{\Omega} d^{d} x \sum_{k=1}^{D}\left[\nabla \phi_{k}(x)\right]^{2}\right]
$$

and a complex amplitude $\mathcal{A}_{V}(\phi)$ :

$$
\mathcal{A}_{V}(\phi)=B\left(\left.\phi\right|_{\partial \Omega}\right) \exp \left\{-\int_{\Omega} d^{d} x V[\phi(x)]\right\}
$$

yields

$$
|V, B, \alpha\rangle=\int d \mu(\phi) \mathcal{A}_{V}(\phi)|\alpha(\phi)\rangle
$$

where $|\alpha(\phi)\rangle=\exp \left\{\int_{\Omega} d^{d} x \alpha[\phi(x)] \psi^{\dagger}(x)\right\}|0\rangle$ is an unnormalized field-coherent state. Hence, just like CMPSs in dimension 1, CTNSs are a generalization of field-coherent states. The latter are obtained, e.g., if $\mathcal{A}_{V}(\phi)$ is only nonzero for a given $\phi$ (for an infinitely deep $V$ ) or, in the homogeneous case, if $\alpha$ is constant.

\section{2. $N$-particle wave function}

A generic state $|\Psi\rangle$ in the bosonic Fock space $\mathcal{F}\left[L^{2}\left(\mathbb{R}^{d}, \mathbb{C}\right)\right]$ can be expanded into a sum of $n$ particle wave functions $\varphi_{n}$ :

$$
|\Psi\rangle=\sum_{n=0}^{+\infty} \int_{\Omega^{n}} d x_{1} \ldots d x_{n} \frac{\varphi_{n}\left(x_{1}, \ldots, x_{n}\right)}{n !} \psi^{\dagger}\left(x_{1}\right) \ldots \psi^{\dagger}\left(x_{n}\right)|0\rangle,
$$

where $\varphi_{n}$ is a completely symmetric function of its coordinates. Simply expanding the exponential of Eq. (1) gives, for the CTNS $|V, B, \alpha\rangle$,

$\varphi_{n}\left(x_{1}, \ldots, x_{n}\right)=\int d \mu(\phi) \mathcal{A}_{V}(\phi) \alpha\left[\phi\left(x_{1}\right)\right] \ldots \alpha\left[\phi\left(x_{n}\right)\right]$.

It provides an equivalent definition of the CTNS.

\section{Correlation functions}

A state is also fully characterized by its (equal time) correlation functions. To compute them, we first introduce the generating functionals for real sources $j^{\prime}, j$ :

$\begin{aligned} & \mathcal{Z}_{j^{\prime}, j}=\frac{\left\langle V, B, \alpha\left|\exp \left(\int_{\Omega} j^{\prime} \cdot \psi^{\dagger}\right) \exp \left(\int_{\Omega} j \cdot \psi\right)\right| V, B, \alpha\right\rangle}{\langle V, B, \alpha \mid V, B, \alpha\rangle}, \\ & \tilde{\mathcal{Z}}_{j^{\prime}, j}=\frac{\left\langle V, B, \alpha\left|\exp \left(\int_{\Omega} j \cdot \psi\right) \exp \left(\int_{\Omega} j^{\prime} \cdot \psi^{\dagger}\right)\right| V, B, \alpha\right\rangle}{\langle V, B, \alpha \mid V, B, \alpha\rangle} .\end{aligned}$

They generate the normal-ordered and anti-normal-ordered correlation functions, respectively. For example, it is straightforward to verify that

$$
\left\langle\psi^{\dagger}(x) \psi(y)\right\rangle:=\frac{\left\langle V, B, \alpha\left|\psi^{\dagger}(x) \psi(y)\right| V, B, \alpha\right\rangle}{\langle V, B, \alpha \mid V, B, \alpha\rangle}
$$

$$
=\left.\frac{\delta}{\delta j^{\prime}(x)} \frac{\delta}{\delta j(y)} \mathcal{Z}_{j^{\prime}, j}\right|_{j, j^{\prime}=0} .
$$

Using the formula for the overlap of (unnormalized) fieldcoherent states,

$$
\langle\beta \mid \alpha\rangle=\exp \left(\int_{\Omega} d x \beta^{*}(x) \alpha(x)\right)
$$

and writing $\mathcal{N}=\langle V, B, \alpha \mid V, B, \alpha\rangle$, we get

$$
\begin{aligned}
\tilde{\mathcal{Z}}_{j^{\prime}, j}= & \frac{1}{\mathcal{N}} \int d \mu\left(\phi^{\prime}\right) d \mu(\phi) B(\phi) B^{*}\left(\phi^{\prime}\right) \\
& \times \exp \left\{-\int_{\Omega} V^{*}\left[\phi^{\prime}\right]+V[\phi]-\left(\alpha^{*}\left[\phi^{\prime}\right]+j\right)\left(\alpha[\phi]+j^{\prime}\right)\right\} .
\end{aligned}
$$

We observe an important fact, which is that the function $\alpha$ appears squared; hence, an $\alpha$ quadratic in the field already brings non-Gaussianities. To compute $\mathcal{Z}_{j^{\prime}, j}$, one applies the Baker-Campbell-Hausdorff (BCH) formula to Eq. (8) to push annihilation operators to the right and get back to a computation of field-coherent state overlaps. We obtain

$$
\begin{aligned}
\mathcal{Z}_{j^{\prime}, j}= & \frac{1}{\mathcal{N}} \int d \mu\left(\phi^{\prime}\right) d \mu(\phi) B(\phi) B^{*}\left(\phi^{\prime}\right) \\
& \times \exp \left\{-\int_{\Omega} V^{*}\left[\phi^{\prime}(x)\right]+V[\phi]-\alpha^{*}\left[\phi^{\prime}\right] \cdot \alpha[\phi]\right. \\
& \left.-j \alpha[\phi]-j^{\prime} \cdot \alpha^{*}\left[\phi^{\prime}\right]\right\},
\end{aligned}
$$

hence the same as Eq. (12) but for the removal of the product $j \cdot j^{\prime}$, which is responsible for the divergent equal point contributions upon functional differentiation. 


\section{B. Operator representation}

\section{State definition}

We now provide an equivalent operator representation of CTNSs. For simplicity, we restrict ourselves to domains of $\mathbb{R}^{d}$ that can be split into Cartesian products $\Omega=$ $[-T / 2, T / 2] \times S$, where $\partial S=\varnothing$. This restriction is not strictly necessary but substantially simplifies the definition.

Definition 2 (alternative operator formulation). - For a domain $\Omega$ that can be written as a Cartesian product $\Omega=[-T / 2, T / 2] \times S$, we write $x=(\tau, \mathbf{x})$, where $\tau \in$ $[-T / 2, T / 2]$ and $\mathbf{x} \in S$. A CTNS is then defined as

$$
\begin{aligned}
& |V, B, \alpha\rangle \\
& =\operatorname{tr}\left[\hat { B } \mathcal { T } \operatorname { e x p } \left(-\int_{-T / 2}^{T / 2} d \tau \int_{S} d \mathbf{x} \sum_{k=1}^{D}\left\{\frac{\left[\hat{\pi}_{k}(\mathbf{x})\right]^{2}}{2}+\frac{\left[\nabla \hat{\phi}_{k}(\mathbf{x})\right]^{2}}{2}\right\}\right.\right. \\
& \left.\left.\quad+V[\hat{\phi}(\mathbf{x})]-\alpha[\hat{\phi}(\mathbf{x})] \psi^{\dagger}(\tau, \mathbf{x})\right)\right]|0\rangle
\end{aligned}
$$

where $\mathcal{T}$ is the $\tau$-ordering operator and $\hat{\phi}_{k}(\mathbf{x})$ and $\hat{\pi}_{k}(\mathbf{x})$ are $k$-independent canonically conjugated pairs of (auxiliary) field operators: $\left[\hat{\phi}_{k}(\mathbf{x}), \hat{\phi}_{l}(\mathbf{y})\right]=0,\left[\hat{\pi}(\mathbf{x})_{k}, \hat{\pi}_{l}(\mathbf{y})\right]=0$, and $\left[\hat{\phi}_{k}(\mathbf{x}), \hat{\pi}_{l}(\mathbf{y})\right]=i \delta_{k, l} \delta^{d-1}(\mathbf{x}-\mathbf{y})$. These operators act on $\mathcal{H}_{\text {aux }}=\mathcal{F}\left[L^{2}(S)\right]^{D}$, i.e., $D$ copies of a bosonic Fock space on a $d-1$-dimensional space. The trace is taken over this auxiliary Hilbert space. As before, $V$ and $\alpha$ may depend on $\mathbf{x}$ and $\tau$.

The operator $\hat{B}$ acts on $\mathcal{H}_{\text {aux }}$ and fixes the boundary conditions; e.g., $\hat{B}=\mathbb{1}$ encodes periodic boundary conditions on the coordinate $\tau$. Another natural option is to take $\hat{B}=\mid$ in $\rangle\langle$ out $|$, which corresponds to the situation in Fig. 2 .

Definitions 1 and 2 are equivalent for this subclass of domains. The proof is straightforward. One just applies the techniques of standard quantum field theory (QFT) textbooks to go from operator to functional integral representations with $\tau=i t$ (see, e.g., Refs. [38,39]). Mainly, one discretizes the $\tau$-ordered product in Eq. (14) into a finite product of terms. One then inserts resolutions of the

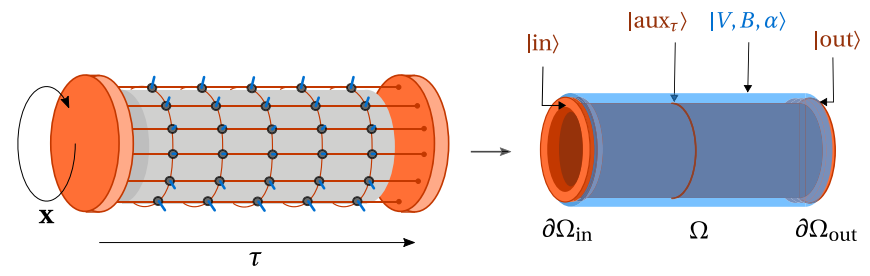

FIG. 2. Operator representation. Tensor networks in $d \geq 2$ can be defined through an auxiliary dynamics in $d-1$ dimensions. In the continuum, the physical $d$-dimensional quantum field $|V, B, \alpha\rangle$ is obtained through a joint nonunitary evolution [Eq. (14)] with an auxiliary $d$-1-dimensional quantum field $\left|\operatorname{aux}_{\tau}\right\rangle$. identity in the field basis $|\phi\rangle$ at every time step $\Delta \tau$ and writes each resulting overlap in the conjugate momentum basis $|\pi\rangle$. Going back to the continuum limit yields a phase space functional integral which reduces to the formula of Eq. (1) upon Gaussian integration of the conjugate momenta $\pi$.

The boundary operator of Eq. (14) is related to the boundary functional of Eq. (1) by

$$
B(\phi)=\left\langle\phi_{\text {in }}|\hat{B}| \phi_{\text {out }}\right\rangle,
$$

where $\left|\phi_{\text {out }}\right\rangle$ and $\left|\phi_{\text {in }}\right\rangle$ are eigenstates of the auxiliary field operators $\hat{\phi}(\mathbf{x})$. The auxiliary field $\phi$ decomposes into $\phi=\phi_{\text {in }}+\phi_{\text {out }}$, where $\phi_{\text {in }}$ (respectively, $\phi_{\text {out }}$ ) has support on $\partial \boldsymbol{\Omega}_{\text {in }}$ (respectively, $\partial \boldsymbol{\Omega}_{\text {out }}$ ) with $\partial \boldsymbol{\Omega}=\partial \boldsymbol{\Omega}_{\text {in }} \cup \partial \boldsymbol{\Omega}_{\text {out }}$.

As before, we propose a repackaging of formula (14). Introducing the Hamiltonian density $\mathcal{H}(\mathbf{x})=\mathcal{H}_{0}(\mathbf{x})+$ $V[\hat{\phi}(\mathbf{x})]$ with $\mathcal{H}_{0}(\mathbf{x})=\sum_{k=1}^{D}\left\{\left(\left[\hat{\pi}_{k}(\mathbf{x})\right]^{2}+\left[\nabla \hat{\phi}_{k}(\mathbf{x})\right]^{2}\right) / 2\right\}$ yields

$$
\begin{aligned}
|V, B, \alpha\rangle= & \operatorname{tr}\left[\hat { B } \mathcal { T } \operatorname { e x p } \left(-\int_{-T / 2}^{T / 2} d \tau \int_{S} d \mathbf{x} \mathcal{H}(\mathbf{x})\right.\right. \\
& \left.\left.-\alpha[\hat{\phi}(\mathbf{x})] \psi^{\dagger}(\tau, \mathbf{x})\right)\right]|0\rangle .
\end{aligned}
$$

This equation is a straightforward extension of the CMPS definition [33] (recalled in Sec. IV D) with $\hat{Q} \sim-\mathcal{H}(\mathbf{x})$ and $\hat{R} \sim \alpha[\hat{\phi}(\mathbf{x})]$.

\section{2. $N$-particle wave function}

The $N$-particle wave function $\varphi_{n}$ defined in Eq. (6) can also be computed in the operator representation. For $-T / 2<\tau_{1}<\cdots<\tau_{n}<T / 2$, we get, expanding the $\tau$-ordered exponential (14) into an infinite product,

$\varphi_{n}=\operatorname{tr}\left[\hat{B} \hat{G}_{T / 2, \tau_{n}} \hat{\alpha}\left(x_{n}\right) \hat{G}_{\tau_{n}, \tau_{n-1}} \hat{\alpha}\left(x_{n-1}\right) \ldots \hat{\alpha}\left(x_{1}\right) \hat{G}_{\tau_{1}, T / 2}\right]$

with $\quad \hat{\alpha}\left(x_{n}\right)=\alpha\left[\hat{\phi}\left(\mathbf{x}_{n}\right)\right] \quad$ and $\quad \hat{G}_{u, v}=\mathcal{T} \exp \left[-\int_{v}^{u} d \tau \times\right.$ $\int_{S} d \mathbf{x} \mathcal{H}(\mathbf{x})$ ]. As for CMPSs [33], we interpret $\hat{G}$ as a propagator and $\hat{\alpha}$ as a scattering matrix creating a particle. It is the very specific form of $\mathcal{H}$ and, hence, of $\hat{G}$ that is responsible for the Euclidean symmetries of the resulting state. Generalizing CMPSs starting directly from Eq. (17) would make it hard to guess an appropriate expression for $\hat{G}$.

Note that Eq. (17) amounts to taking as the wave function a correlation function of auxiliary quantum fields, which is similar in spirit with the Moore-Read states [40] used for Hall physics or with the infinite matrix product states [41] used for critical spin chains.

\section{Correlation functions}

Finally, we provide an expression for the generating functionals $\quad \mathcal{Z}_{j^{\prime}, j} \quad \tilde{\mathcal{Z}}_{j^{\prime}, j}$ in the operator representation. 
Exploiting the operator definition of the CTNS (14) and expanding the $\tau$-ordered exponential into an infinite product of infinitesimal exponentials, we get

$$
\tilde{\mathcal{Z}}_{j^{\prime}, j}=\operatorname{tr}\left[B \otimes B^{*} \mathcal{T} \exp \left(\int_{-T / 2}^{T / 2} \mathbb{T}_{j^{\prime} j}\right)\right]
$$

with the transfer matrix (with sources)

$$
\mathbb{T}_{j^{\prime} j}=\int_{S}-\mathcal{H} \otimes \mathbb{1}-\mathbb{1} \otimes \mathcal{H}^{*}+\left(\alpha[\hat{\phi}]+j^{\prime}\right) \otimes\left(\alpha[\hat{\phi}]^{*}+j\right) .
$$

Using as before the $\mathrm{BCH}$ formula yields

$$
\mathcal{Z}_{j^{\prime} j}=\operatorname{tr}\left[B \otimes B^{*} \mathcal{T} \exp \left\{\int_{-T / 2}^{T / 2}\left(\mathbb{T}_{j^{\prime} j}-\int_{S} j \cdot j^{\prime}\right)\right\}\right] .
$$

The functional derivatives can then be carried explicitly, and one obtains e.g., for $-T / 2<\tau_{2}<\tau_{1}<T / 2$,

$$
\begin{aligned}
\left\langle\psi^{\dagger}\left(x_{1}\right) \psi\left(x_{2}\right)\right\rangle= & \operatorname{tr}\left\{B \otimes B^{*} \cdot \mathcal{M}_{T / 2, \tau_{1}} \cdot\left[\mathbb{1} \otimes \hat{\alpha}^{*}\left(x_{1}\right)\right]\right. \\
& \left.\cdot \mathcal{M}_{\tau_{1}, \tau_{2}} \cdot\left[\hat{\alpha}\left(x_{2}\right) \otimes \mathbb{1}\right] \cdot \mathcal{M}_{\tau_{2},-T / 2}\right\}
\end{aligned}
$$

with the map $\mathcal{M}_{u, v}=\mathcal{T} \exp \left[\int_{v}^{u} \mathbb{T}\right]$ and the transfer matrix $\mathbb{T}:=\mathbb{T}_{00}$. More generally, correlation functions are given by the trace of a succession of propagators $\mathcal{M}$ followed by operator insertions of $\alpha \otimes \mathbb{1}$ (respectively, $\mathbb{1} \otimes \alpha^{*}$ ) in the positions corresponding to $\psi$ (respectively, $\psi^{\dagger}$ ).

\section{LINK WITH DISCRETE TENSOR NETWORK STATES}

\section{A. (Discrete) tensor network states}

We start with a very brief reminder on TNSs, recalling only their elementary definition. For an understanding of their efficiency in representing quantum systems of physical interest, we direct the reader to the relevant literature (e.g., Refs. $[42,43]$, and references therein).

TNSs are variational ansatz for many-body wave functions that take the form of a contraction of local tensors. The simplest example, in spatial dimension $d=1$, is provided by MPSs. For a translation-invariant quantum spin- $1 / 2$ chain with $N$ sites, a generic state reads

$$
|\psi\rangle=\sum_{i_{1}, \ldots, i_{N}=\{-1,1\}^{N}} c_{i_{1}, \ldots, i_{N}}\left|i_{1}\right\rangle \otimes \cdots \otimes\left|i_{N}\right\rangle,
$$

where the wave function $c_{i_{1}, \ldots, i_{N}}$ contains $2^{N}$ complex parameters. A MPS is an economical ansatz for this wave function:

$$
c_{i_{1}, \ldots, i_{n}}=\operatorname{tr}\left[A_{i_{1}} \ldots A_{i_{N}}\right]
$$

where $A_{-1}$ and $A_{1}$ are two $\chi \times \chi$ matrices. These matrices contain the parameters which allow one to vary the state. Their size $\chi$, called the bond dimension, encodes the depth of the variational class and upper bounds the amount of spatial entanglement that can be carried by the state.

The two matrices can be be collected into a 3-index tensor $\left[A_{i}^{k, \ell}\right]_{i= \pm 1}^{k, \ell=1 \ldots \chi}$ written graphically:

$$
A_{i}^{k, \ell}=k \stackrel{i}{\stackrel{d}{0}} \ell,
$$

where $i$ is the physical index and $k$ and $\ell$ are so-called bond indices. Graphically, the corresponding wave function $c$ can be written

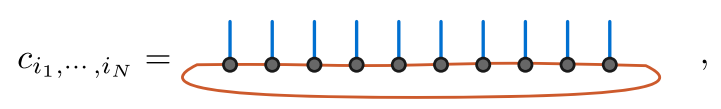

where joint legs of the tensor $A$ denote a summation on the corresponding index, associated with the matrix multiplication and subsequent trace in Eq. (23). This representation makes it natural to generalize matrix product states to PEPSs [13] in arbitrary dimensions, e.g., in $d=2$ for $N \times N$ sites:

$$
c_{i_{1}, \cdots, i_{N^{2}}}=\frac{0 b \mathrm{do}}{0 b^{2}}
$$

This latter object, in general $d$, is what the CTNS defined in Eqs. (1) or (14) aims to extend to the continuum.

\section{B. Constructing CTNSs}

Our objective is to show how CTNSs can be obtained from a limit of a discrete TNS. To motivate this discrete ansatz, we first provide heuristics for why its main characteristics seem to be required. Mainly, we aim to justify (i) why an infinite bond dimension is needed and (ii) why the trivial tensor around which we expand is of the form we postulate.

The first point is a scaling argument. We ask for a strong notion of continuum limit: We require the discrete tensor to be approximately stable by fine graining to the UV. Namely, the discrete ansatz needs to be (at least approximately) expressible as a contraction of tensors with the same form but different parameters. Each blocking multiplies the physical dimension by $2^{d}$, which is why in the continuum limit one obtains a field theory on the physical degrees of freedom. But each blocking also multiplies the bond dimension by $2^{d-1}$ (see Fig. 3). Hence, for $d>1$, the bond dimension is increased when zooming out and decreased when zooming in. The only way to make the class of states considered approximately stable is for the bond dimension to be infinite. Notice in this respect that the $d=1$ case allows finite bond dimensions even in the 


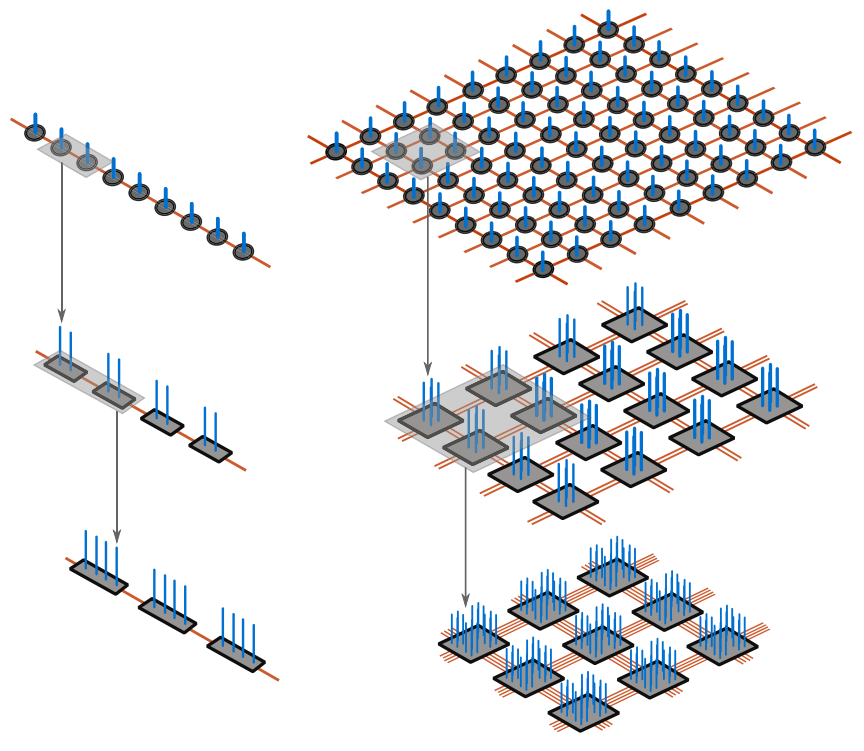

FIG. 3. Tensor blocking. In $d=1$, blocking does not increase the bond dimension. In $d=2$, going from the UV to the IR doubles the bond dimension at each blocking. Hence, flowing the other way, from IR to UV, one reaches a trivial bond dimension after a finite number of iterations unless the initial bond dimension is infinite.

continuum limit $[33,34]$. It will be important to see if this peculiarity can be recovered in some appropriate limit in Sec. IV D.

Note that our argument in favor of an infinite bond dimension does not imply that a discrete tensor network state with a finite bond dimension could not behave, at distances sufficiently large compared to the lattice spacing, like a CTNS. Rather, our argument shows that any simple space discretization of a CTNS in $d \geq 2$ into a TNS will have an infinite bond dimension, even for an arbitrarily small lattice spacing. As in Ref. [35], it could also be that a proper choice of boundary conditions would constrain the tensor contraction on a finite-dimensional subspace, despite an apparent infinite bond dimension.

We now need to discuss more precisely the form of the elementary tensor. For notational simplicity, we now assume that an elementary tensor $\hat{T}$ to be contracted,

$$
\hat{T}:=\gamma<
$$

is a vector in its bond indices but an operator acting on the vacuum in the physical space, namely,

$$
\begin{aligned}
\mid \text { physical state }\rangle & =\text { contraction }\{\text { network of } \hat{T}\}|0\rangle \\
& =
\end{aligned}
$$

To obtain a continuum limit, the crucial choice lies in the elementary "trivial tensor," acting as the identity on the vacuum, and around which to expand:

$$
\hat{T}=\hat{T}^{(0)}+\varepsilon^{d} \times \text { corrections. }
$$

Indeed, it is natural to want the tensor corresponding to zero particle in an elementary cell of the physical space to dominate. It seems that any other choice would preclude the existence of a continuum limit. In $d=1$ dimension, there is only one natural option, which is to take the tensor corresponding to the identity:

$$
\hat{T}^{(0)}=- \text {. }
$$

But, in the same way as for the bond dimension, the situation is a little too trivial in $d=1$ to give a precise hint for higher dimensions. In $d>1$, there are several seemingly natural options which we have to inspect. We discuss the $d=2$ case, but the reasoning holds for any $d \geq 2$. We do not aim to prove that the tensor we ultimately expand around is the only option but rather that other seemingly simpler options bring difficulties.

(1) A naive option is to generalize the identity on the auxiliary bond space by taking $\hat{T}_{i j k l}^{(0)}=\delta_{i j k l}$, that is, to take an elementary tensor corresponding to a Greenberger-Horne-Zeilinger (GHZ) state on the bond indices:

$$
\hat{T}^{(0)}=><.
$$

As will later be manifest, this choice is too brutal and would yield a state with a trivial spatial structure.

(2) Another simple option is to take the identity along a diagonal, e.g.,

$$
\left.\hat{T}^{(0)}=\right\rangle<
$$

This is the choice that stays the closest in spirit with the $d=1$ case. The problem of such a choice (made, e.g., in Ref. [35]) is that it picks a preferred direction and thus makes Euclidean invariance impossible to obtain directly (that is, without analytic continuation).

(3) We may combine the $2^{d-1}$ identity operator along diagonals in a sum, as an attempt to recover the Euclidean invariance lost with the previous choice:

$$
\hat{T}^{(0)}=><+\rightleftharpoons \text {. }
$$

An issue is then that the corresponding tensor contraction contains loops 


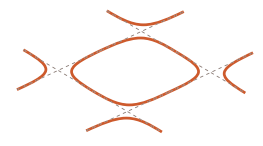

which yield divergent terms as $\operatorname{tr}[\mathbb{1}]=+\infty$ for an infinite bond dimension.

None of the natural options seem to provide a simple Euclidean-invariant continuum limit. Our proposal consists in taking a regularized version of the first possibility, in the form of a "soft" delta:

$$
\hat{T}^{(0)}=3
$$

\section{Discrete ansatz in $d=2$}

The first lesson from the previous sections is that infinite bond dimensions seem to be required. We thus write the bond indices of the elementary tensor as $D$ real numbers. In $d=2$, this means that an elementary tensor has four bond indices $\phi(1), \phi(2), \phi(3)$, and $\phi(4) \in \mathbb{R}^{D}$ :

$$
\hat{T}_{\phi(1), \phi(2), \phi(3), \phi(4)}={ }_{\phi(1)}^{\phi(2)} \succ_{\phi(4)}^{\phi(3)} .
$$

As we mentioned before, the heart of the problem of the continuum limit lies in defining the proper trivial tensor around which to expand. We choose a soft delta:

$$
\begin{aligned}
\hat{T}^{(0)}= & \\
=\exp & \left\{\frac{-1}{2} \sum_{k=1}^{D}\left[\phi_{k}(1)-\phi_{k}(2)\right]^{2}+\left[\phi_{k}(1)-\phi_{k}(4)\right]^{2}\right. \\
+ & {\left.\left[\phi_{k}(3)-\phi_{k}(2)\right]^{2}+\left[\phi_{k}(3)-\phi_{k}(4)\right]^{2}\right\} }
\end{aligned}
$$

This ansatz forces the bond indices to remain close to each other and contributes to the generation of the gradient squared term in the action. Being Gaussian, we also naturally expect its form to be stable. To this "trivial" part, we add local corrections of the order of $\varepsilon^{d}=\varepsilon^{2}$ :

$$
\hat{T}=\hat{T}^{(0)} \exp \left[-\varepsilon^{2} V(\phi) \mathbb{1}+\varepsilon^{2} \alpha(\phi) \psi^{\dagger}(x)\right] .
$$

In this expression, $\phi$ denotes whatever combination of the bond-field indices $\phi(1), \phi(2), \phi(3)$, and $\phi(4)$. The simplest possibility is to take $\phi$ as the average of the bond indices, but it does not matter for the continuum limit. The operator $\psi^{\dagger}(x)$ anticipates the continuum and has commutation relations $\left[\psi(x), \psi^{\dagger}(y)\right]=\left(1 / \varepsilon^{2}\right) \delta_{x, y} \simeq \delta^{2}(x-y)$.

Ignoring the boundary conditions for now, the contraction of the tensors amounts to integrate over all the bond indices:

$$
\begin{aligned}
|V, \alpha\rangle= & \int \prod_{x \in \text { lattice }} \hat{T}(x) \prod_{k=1}^{D} d \phi_{k}\left(x+\frac{\varepsilon}{2} \mathbf{e}_{1}\right) \\
& \times d \phi_{k}\left(x+\frac{\varepsilon}{2} \mathbf{e}_{2}\right)|0\rangle,
\end{aligned}
$$

where $\mathbf{e}_{1}$ and $\mathbf{e}_{2}$ are unit vectors along the two lattice directions and the bond fields are indexed by the points on the links of the lattice where they sit. Writing $u=\left[\left(x^{1}+x^{2}\right) / \sqrt{2}\right]$ and $v=\left[\left(x^{1}-x^{2}\right) / \sqrt{2}\right]$, we see that the differences in Eq. (38) yield

$$
\begin{aligned}
& \prod_{x \in \text { lattice }} \hat{T}(x) \underset{\varepsilon \rightarrow 0}{\simeq} \exp \left\{-\int d^{2} x \frac{\left[\partial_{u} \phi_{k}(x)\right]^{2}+\left[\partial_{v} \phi_{k}(x)\right]^{2}}{2}\right. \\
& \left.+V[\phi(x)]-\alpha[\phi(x)] \psi^{\dagger}(x)\right\} .
\end{aligned}
$$

We recognize the (rotation-invariant) gradient squared term of the continuum definition II A 1. Defining the path integral "measure" as

$$
\mathcal{D} \phi: \simeq \lim _{\varepsilon \rightarrow 0} \prod_{\substack{x \in \text { lattice } \\ k=1 \ldots D}} d \phi_{k}\left(x+\frac{\varepsilon}{2} \mathbf{e}_{1}\right) d \phi_{k}\left(x+\frac{\varepsilon}{2} \mathbf{e}_{2}\right)
$$

finally yields the continuous tensor network state of Eq. (1) up to boundary conditions. To get a state on the physical Hilbert space, the auxiliary fields on the boundary just have to be contracted (or integrated) against a boundary functional, which we write $B$ in Eq. (1).

\section{Discrete ansatz in general $d$}

For $d \geq 3$, the derivation is carried along the same way as before. We just note that there is a small peculiarity in the $d=2$ case because the auxiliary fields are adimensional. To generalize Eq. (38) to higher spatial dimensions $d>2$, one naturally extends the prescription of summing all the differences of the squares of the nearest bond indices $\phi(1), \ldots \phi(2 d)$. But, importantly, to obtain the continuum limit, one needs to multiply this expression by $\varepsilon^{d-2}$, where $\varepsilon$ is the length of the unit cell:

$$
\hat{T}_{\phi(1) \ldots \phi(2 d)}^{(0)}=\exp \left\{-\frac{\varepsilon^{d-2}}{2} \sum_{k=1}^{D} \cdots\right\}
$$

to obtain the integral of a gradient squared in the continuum limit. In $d=2$, the $\varepsilon^{d}$ of the integration measure and $\varepsilon^{-2}$ from the gradient squared cancel each other, and this scaling factor does not appear.

In retrospect, it is clear why deriving the continuum limit by perturbing around the GHZ tensor (32) gives a trivial continuum. It corresponds to putting an infinitely large constant instead of $\varepsilon^{d-2}$ in Eq. (43), an infinite "rigidity" that could not be compensated by locally small terms. 


\section{PROPERTIES}

We now explore the properties of CTNSs that are analogous to those of their discrete counterparts.

\section{A. Stability and expressiveness}

It is first natural to wonder how "big" the class of CTNSs is. It could be, for example, that even for arbitrary large $D$ and arbitrary $V, B$, and $\alpha$, CTNSs spanned only a small sector of the Fock space. As in the discrete, can any state be approximated, even if inefficiently, by a CTNS?

Let us first consider the stability of the CTNS class. The sum of two CTNSs is still a CTNS, provided we are willing to accept singular potentials $V$. More precisely, let $\left|V_{1}, B_{1}, \alpha_{1}\right\rangle$ and $\left|V_{2}, B_{2}, \alpha_{2}\right\rangle$ be two CTNSs with bondfield dimension $D_{1}$ and $D_{2}$. Then we can easily rewrite their sum as a CTNS with bond-field dimension $D_{1}+$ $D_{2}+1$ (although it may, in general, require fewer auxiliary fields). For example, defining the CTNS $\left|W_{\Lambda}, C, \beta\right\rangle$ with

$$
\begin{aligned}
W_{\Lambda}\left(\phi_{1}, \phi_{2}, \tilde{\phi}\right)= & V_{1}\left(\phi_{1}\right) \theta(\tilde{\phi})+V_{2}\left(\phi_{2}\right) \theta(-\tilde{\phi}) \\
& +\Lambda(\tilde{\phi}-1)^{2}(\tilde{\phi}+1)^{2}, \\
C\left(\phi_{1}, \phi_{2}, \tilde{\phi}\right) & =B_{1}\left(\phi_{1}\right) \theta(\tilde{\phi})+B_{2}\left(\phi_{2}\right) \theta(-\tilde{\phi}), \\
\beta\left(\phi_{1}, \phi_{2}, \tilde{\phi}\right) & =\alpha_{1}\left(\phi_{1}\right) \theta(\tilde{\phi})+\alpha_{2}\left(\phi_{2}\right) \theta(-\tilde{\phi}),
\end{aligned}
$$

where $\theta$ is the Heaviside function, we have $\left|W_{\infty}, C, \beta\right\rangle \propto$ $\left|V_{1}, B_{1}, \alpha_{1}\right\rangle+\left|V_{2}, B_{2}, \alpha_{2}\right\rangle$. Indeed, when $\Lambda$ is sent to infinity, the auxiliary field $\tilde{\phi}$ becomes a "bit" taking values \pm 1 digitally splitting the functional integral into two contributions $\int \mathcal{D} \tilde{\phi} \simeq \sum_{\tilde{\phi} \equiv \pm 1}$, where each term of the sum gives the two initial states.

The expressiveness of CTNSs is then easy to assess, following the same technique as for CMPSs [35]. Taking $\alpha[x, \phi(x)]=f(x)$ and $V=a / \operatorname{Vol}(\Omega)$, we obtain any field-coherent state with any complex weight $e^{-a}|f\rangle$. Using the stability result, one can construct arbitrary linear combinations of such field-coherent states which are dense in Fock space; hence, one can get arbitrarily close to any state in the Fock space. With this construction, the bond-field dimension grows at each addition of coherent states.

Actually, using larger bond-field dimensions is only a convenience, and, provided $V$ and $\alpha$ are arbitrary, a CTNS can approximate any state in the Fock space with $D=1$. Let us consider a sum of coherent states $|\Psi\rangle=$ $\sum_{j=1}^{m} e^{-a_{i}}\left|f_{i}\right\rangle$. This sum can be approximated by the CTNS $\left|V_{\Lambda}, 1, \alpha\right\rangle$ with

$$
\begin{aligned}
V_{\Lambda}[x, \phi(x)]= & -\Lambda \mathbf{1}_{[-1 / 2, m+1 / 2]}[\phi(x)] \cos [2 \pi \phi(x)] \\
& +\sum_{j=1}^{m} a_{j} \mathbf{1}_{[j-1 / 2, j+1 / 2]}[\phi(x)],
\end{aligned}
$$

$$
\alpha[x, \phi(x)]=\sum_{j=1}^{m} \mathbf{1}_{[j-1 / 2, j+1 / 2]}[\phi(x)] f_{j}(x),
$$

where $\mathbf{1}_{A}[x]=1$ if $x \in A$ and 0 otherwise. Indeed, when $\Lambda \rightarrow+\infty$, the auxiliary field is forced to sit on one of the $m$ minima of the potential, which each have a complex weight $e^{-a_{j}}$. To each of these $m$ possible values of the field, the term in $\alpha$ associates a different coherent state. Hence, one can approximate $|\Psi\rangle$ with arbitrary precision and, hence, all states in the Fock space.

Allowing larger values of $D$ remains useful if $V$ and $\alpha$ are restricted in some way, e.g., to being polynomials with a fixed degree. In that case, being able to take a larger bondfield dimension $D$ substantially increases the expressiveness of a CTNS subclass. Gaussian CTNSs (see Sec. VA) provide such an illustration.

\section{B. Gauge transformation}

Different choices of $V, B$, and $\alpha$ can generate the same state. This fact is to be expected: In the discrete, the map between an elementary tensor and a many-body wave function is not injective, either. Understanding the transformations between tensors generating the same state is fundamental in the theory of TNSs, especially for the classification of symmetry-protected and topological phases. It is thus natural to ask the same question for CTNSs following the discrete construction.

\section{Intuition from the discrete}

In the discrete, there exists an important subclass of transformations one can apply on the bond indices of an elementary tensor and that leave the state invariant. For example, in $d=2$, the transformation (sometimes called gauge transformation)

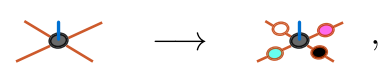

where $\sigma^{\sigma}=>$ and $a_{2}=\backslash$ gives the same contracted state up to new boundary terms (that vanish on a torus). Such transformations prove central to classifying topological phases of matter with discrete tensor networks. We would thus like to find an analog in the continuum.

For an infinite bond dimension, the equivalent of an invertible linear transformation acting on discrete indices is a linear operator $G$ acting on functions of $D$ real variables (the auxiliary field):

$$
\mathrm{G} \cdot \varphi(\phi)=\int d^{D} \tilde{\phi} \mathrm{G}(\phi, \tilde{\phi}) \varphi(\tilde{\phi}) .
$$

A $G$ that is too generic typically destroys the continuum limit when the corresponding gauge transformation (49) is applied on the elementary tensor. The main difficulty is to 
know what subset of operators to look at. For simplicity, we restrict ourselves to the case $D=1$, from which the general case is easily deduced.

A first option is to consider the subset of diagonal transformations. Let $F$ and $G$ be two operators acting diagonally:

$$
\begin{aligned}
\mathrm{G} \cdot \varphi(\phi) & =\mathrm{g}(\phi) \varphi(\phi), \\
\mathrm{F} \cdot \varphi(\phi) & =\mathrm{f}(\phi) \varphi(\phi) .
\end{aligned}
$$

Acting on an elementary discrete tensor in the same way as in Eq. (49) with F and G simply changes the integration measure:

$$
d \phi(1) \ldots d \phi(4) \rightarrow \frac{f[\phi(1)] g[\phi(4)]}{f[\phi(3)] g[\phi(2)]} d \phi(1) \ldots d \phi(4) .
$$

If this change of measure is too general, there will be no continuum limit. A natural choice, preserving the continuum, is to take

$$
\begin{aligned}
\mathfrak{f}(\phi) & =\exp \left[-\varepsilon^{d-1} \mathfrak{f}(\phi)\right], \\
\mathbf{g}(\phi) & =\exp \left[-\varepsilon^{d-1} \mathfrak{g}(\phi)\right] .
\end{aligned}
$$

In the continuum limit, such a choice put in Eq. (53) yields

$$
\mathcal{D} \phi \rightarrow \mathcal{D} \phi \exp \left[\int d^{2} x \nabla \cdot\left(\begin{array}{c}
\mathfrak{f}[\phi(x)] \\
\mathfrak{g}[\phi(x)]
\end{array}\right)\right]
$$

hence, this choice adds a pure divergence term into the CTNS definition which can be transformed into a boundary term thanks to Stokes' theorem, which is exactly what a gauge transformation should do.

This very special choice of operators does not exhaust the infinitesimal transformations compatible with the existence of a continuum limit. However, we conjecture that all discrete gauge transformations of the form of Eq. (49) that
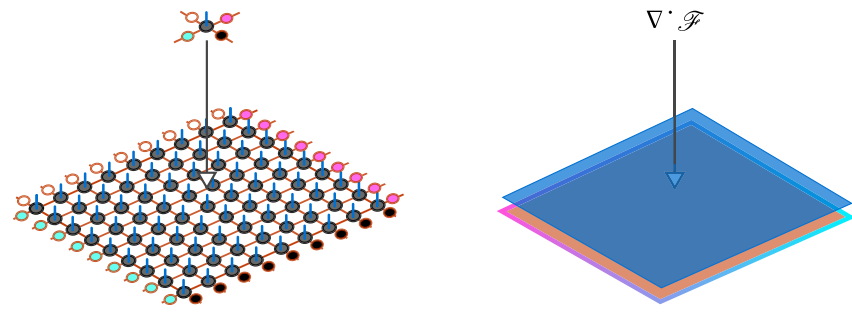

FIG. 4. Gauge transformations. In the discrete case (left), transforming the elementary tensor as in Eq. (49) has a nontrivial result on the boundary only. In the continuum (right), the transformation of the elementary tensor is equivalent to the addition of a pure divergence term for the auxiliary fields in the bulk, which can then be integrated into a boundary condition. preserve the continuum limit ultimately give rise to pure divergence terms as well. In any case, this discussion of the discrete setting is but a motivation for the introduction of (some) continuous gauge transformations of CTNSs (see Fig. 4).

\section{Continuum description}

The previous inquiries motivate the following proposition, which is at the same time a definition of a certain class of gauge transformations for CTNSs.

Proposition 1 (gauge transformation). - Let $\mathcal{F}[x, \phi(x)]$ be an arbitrary vector field in $\Omega$. If $\Omega$ has no boundary, the CTNS $|V, \alpha\rangle$ is left unchanged by the gauge transformation:

$$
V(\phi) \rightarrow V(\phi)-\nabla \cdot \mathcal{F}[x, \phi(x)] .
$$

The proof is trivial and is just a direct application of Stokes' theorem. More generally, if $\Omega$ has a boundary $\partial \Omega$, the gauge transformation (57) adds a boundary term to the measure:

$B(\phi) \rightarrow B(\phi) \exp \left\{\oint_{\partial \Omega} d^{d-1} x \mathcal{F}[x, \phi(x)] \cdot \mathbf{n}(x)\right\}$,

where $\mathbf{n}(x)$ is the unit vector normal to $\partial \Omega$ in $x$. Gauge transformations of CTNSs thus have a straightforward geometric interpretation.

\section{Tensor rescaling}

Our objective is now to relate different tensor network descriptions of the same state at different scales [14,44]. More precisely, considering a correlation function for a state parametrized by a tensor $T(1)$ in the thermodynamic limit:

$$
C\left(x_{1}, \ldots, x_{n}\right)=\left\langle T(1)\left|\mathcal{O}\left(x_{1}\right) \ldots \mathcal{O}\left(x_{n}\right)\right| T(1)\right\rangle,
$$

the objective is to find a tensor $T(\lambda)$ of new parameters such that

$$
C\left(\lambda x_{1}, \ldots, \lambda x_{n}\right) \propto\left\langle T(\lambda)\left|\mathcal{O}\left(x_{1}\right) \ldots \mathcal{O}\left(x_{n}\right)\right| T(\lambda)\right\rangle .
$$

Naturally, in the discrete, this relation is at best approximate.

For the CTNS of Definition 1, we can write the flow $V(\lambda), \alpha(\lambda)$ exactly, following the rather standard dimensional analysis of ordinary QFT. As such, we introduce new creation and annihilation operators $\tilde{\psi}^{\dagger}(x)=\lambda^{d / 2} \psi^{\dagger}(x \lambda)$ and $\tilde{\psi}(x)=\lambda^{d / 2} \psi(x \lambda)$. They indeed verify the standard commutation relations $\left[\tilde{\psi}(x), \tilde{\psi}^{\dagger}(x)\right]=\delta(x-y)$. These new operators relate correlation functions at different scales: 


$$
\begin{aligned}
C\left(\lambda x_{1}, \ldots, \lambda x_{n}\right) & :=\left\langle\psi^{\dagger}\left(\lambda x_{1}\right) \ldots \psi\left(\lambda x_{n}\right)\right\rangle \\
& =\lambda^{-n d / 2}\left\langle\tilde{\psi}^{\dagger}\left(x_{1}\right) \ldots \tilde{\psi}^{\dagger}\left(x_{n}\right)\right\rangle,
\end{aligned}
$$

where the $\lambda^{-n d / 2}$ factor just comes from the fact that the operators we introduce have a dimension. We just have to rewrite $|V, \alpha\rangle$ as a function of the new creation and annihilation operators to relate the different scales. To achieve this rescaling, we change the position variable introducing $u=x / \lambda$. The free field measure now reads

$$
\begin{aligned}
d \mu(\phi) & =\mathcal{D} \phi \exp \left(-\frac{1}{2} \int d^{d} u \lambda^{d-2} \nabla \phi_{k}(u \lambda) \cdot \nabla \phi_{k}(\lambda u)\right) \\
& \propto \mathcal{D} \tilde{\phi} \exp \left(-\frac{1}{2} \int d^{d} u \nabla \tilde{\phi}_{k}(u) \cdot \nabla \tilde{\phi}_{k}(u)\right) \\
& =d \mu(\tilde{\phi}),
\end{aligned}
$$

with $\tilde{\phi}(u)=\lambda^{[(d-2) / 2]} \phi(\lambda u)$. This rescaling gives

$$
\begin{aligned}
|V, \alpha\rangle_{\psi}= & \int d \mu(\tilde{\phi}) \exp \left\{-\int d^{d} u \lambda^{d} V\left[\lambda^{(2-d) / 2} \tilde{\phi}(u)\right]\right. \\
& \left.-\lambda^{d / 2} \alpha\left[\lambda^{(2-d) / 2} \tilde{\phi}(u)\right] \tilde{\psi}^{\dagger}(u)\right\}|0\rangle \\
= & \left|\lambda^{d} V\left[\lambda^{(2-d) / 2} \cdot\right], \lambda^{d / 2} \alpha\left[\lambda^{(2-d) / 2} \cdot\right]\right\rangle_{\tilde{\psi}} .
\end{aligned}
$$

This result allows us to discuss the IR behavior of CTNSs in terms of relevant, irrelevant, and marginal couplings. To this end, we informally expand $V$ and $\alpha$ in powers $p$ of the fields $\phi$ and analyze the terms of each degree separately. The corresponding coupling dimensionality is $\Delta=d+$ $[(2-d) / 2] p$ for terms in $V$ and $\Delta=(d / 2)+[(2-d) / 2] p$ for $\alpha$. Consequently,

For $d=2$, all powers of the field in $V$ and $\alpha$ yield relevant couplings. There are no irrelevant couplings.

For $d=3$, the powers $p=1,2,3,4,5$ of the field in $V$ yield relevant $\Delta>0$ couplings. The power $p=6$ is marginal in $V$. For $\alpha$, the powers $p=1,2$ are relevant and $p=3$ is marginal. All other powers are irrelevant. Relevant powers will dominate the behavior of CTNS correlation functions in the IR and actually be the only ones allowed if the CTNS description is aimed to hold at all scales in the non-Gaussian case (see Sec. V B).

\section{Recovering continuous matrix product states}

Continuous TNSs should reduce to CMPSs in an appropriate limit, which is an important property to check to demonstrate that our ansatz is a natural extension of CMPSs to $d \geq 2$.

\section{Compactification}

A CMPS of a quantum field defined on a space interval of length $T$ is parametrized by three $(\chi \times \chi)$ matrices $\hat{Q}, \hat{B}$, and $\hat{R}$ and is defined [33,34] as

$|Q, B, R\rangle=\operatorname{tr}\left\{\hat{B} \mathcal{T} \exp \left[\int_{-T / 2}^{T / 2} d \tau \hat{Q}+\hat{R} \otimes \psi^{\dagger}(\tau)\right]\right\}|0\rangle$.

To obtain a CMPS, we could directly instantiate our CTNS ansatz with $d=1$, e.g., using its functional integral form (1). However, as we mentioned before, the $d=1$ case is quite peculiar compared to other dimensions, and so it is nice to see it can also be immediately obtained from a general $d$ case where all dimensions but one are taken to be very small.

Indeed, consider a domain of the form $\Omega=$ $[-T / 2, T / 2] \times S$, where $S$ is a $d-1$-dimensional torus of length $\ell$ in all $d-1$ directions. Expanding the auxiliary fields $\phi$ of Eq. (1) in Fourier modes on this $d-1$ torus $S$ and taking the limit $\ell \rightarrow 0$ yields a functional integral in which only the field zero mode on $S$ survives. Hence, one obtains a functional integral of the form

$$
\begin{aligned}
|V, B, \alpha\rangle= & \int \mathcal{D} \phi B(\phi) \exp \left\{-\int_{-T / 2}^{T / 2} d x \frac{1}{2} \sum_{k=1}^{D}\left[\partial_{x} \phi_{k}(x)\right]^{2}\right. \\
& \left.+V[\phi(x)]-\alpha[\phi(x)] \psi^{\dagger}(x)\right\}|0\rangle
\end{aligned}
$$

where the one-dimensional auxiliary field $\phi$ is the zero mode (on the shrunk torus $S$ ) of the initial $d$-dimensional auxiliary field. It is what one would have obtained by fixing immediately $d=1$ in Eq. (1). In operator form, Eq. (69) becomes:

$$
\begin{aligned}
|V, B, \alpha\rangle= & \operatorname{tr}\left[\hat { B } \mathcal { T } \operatorname { e x p } \left(-\int_{-T / 2}^{T / 2} d \tau \sum_{k=1}^{D} \frac{\hat{P}_{k}^{2}}{2}\right.\right. \\
& \left.\left.+V[\hat{X}]-\alpha[\hat{X}] \psi^{\dagger}(\tau)\right)\right]|0\rangle,
\end{aligned}
$$

where $\hat{P}_{k}$ and $\hat{X}_{k}$ are canonically conjugated pairs ( $D$ zerodimensional quantum fields). This is already a CMPS with $-\hat{Q}=\sum_{k=1}^{D}\left(\hat{P}_{k}^{2} / 2\right)+V[\hat{X}]$ and $\hat{R}=\alpha[\hat{X}]$. However, the bond Hilbert space is now that of $D$ particles in one dimension or one particle in $D$ dimensions, and hence $\chi=+\infty$.

\section{Bond-dimension quantization}

To obtain a genuine CMPS (with a finite bond dimension) from a $d=1$ CTNS defined by Eq. (70), we need to choose a specific potential effectively reducing the Hilbert 
space dimensionality. The intuition is quite clear: Take a potential with deep minima.

Let us take a potential with $D$ deep minima $m_{k}$ on the vertices closest to 0 of an hypercube, i.e., $m_{1}=(1,0, \ldots, 0)$, $m_{2}=(0,1,0, \ldots, 0), \ldots$, and $m_{D}=(0, \ldots, 0,1)$. The effective dynamics is now restricted to a $D$-dimensional Hilbert space spanned by $\left|m_{k}\right\rangle$ corresponding to wave packets localized around each minima. In this reduced Hilbert space, the minima are coupled by tunneling. Because of the geometrical configuration we consider, the minima can all be connected by independent saddle points, and, hence, the effective coupling between the minima can be chosen freely. With this freedom, we can obtain any $D \times D$ complex matrix $Q$ of standard CMPSs by adjusting the value of the $D^{2}$ saddle points of $V$.

The $R$ matrix is fixed in the same way. The values of $\alpha[X]$ on the minima $\left|m_{k}\right\rangle$ of the potential fix the diagonal coefficients of $R$, and the value on the saddle point connecting $\left|m_{k_{1}}\right\rangle$ and $\left|m_{k_{2}}\right\rangle$ fixes the nondiagonal terms $R_{k_{1}, k_{2}}$.

Hence, not only can CTNSs reduce to CMPSs when $d=1$ (or when $d-1$ dimensions are small) for a specific choice of potential, but actually all (bosonic) CMPSs can be obtained this way. In this context, the bond-field dimension $D$ reduces to the usual bond dimension $\chi$.

\section{COMPUTATIONS}

To carry computations with CTNSs, one could of course rediscretize them and use the standard TNS algorithms. We now mention techniques relying only on the continuum limit.

\section{A. Gaussian states}

There exists a subclass of CTNSs for which all quantities of interest can be computed exactly: Gaussian CTNSs.

Definition 3 (Gaussian CTNSs). - A CTNS is said to be Gaussian if the functions $V$ and $\alpha$ are, respectively, at most quadratic and affine in the auxiliary field:

$$
\begin{gathered}
V(x, \phi)=V^{(0)}(x)+V_{k}^{(1)}(x) \phi_{k}+\frac{1}{2} V_{k \ell}^{(2)}(x) \phi_{k} \phi_{\ell}, \\
\alpha(x, \phi)=\alpha^{(0)}(x)+\alpha_{k}^{(1)}(x) \phi_{k} .
\end{gathered}
$$

Naturally, a Gaussian CTNS is also a Gaussian state in the usual sense of the term. More precisely, for a Gaussian CTNS, $\mathcal{Z}_{j^{\prime}, j}$ and $\tilde{\mathcal{Z}}_{j^{\prime}, j}$ are manifestly Gaussian functionals. Let us compute $\mathcal{Z}_{j^{\prime}, j}$ in the translation-invariant case $\Omega=\mathbb{R}^{d}$. Inserting Definition 3 into Eq. (13) yields

$$
\begin{aligned}
\mathcal{Z}_{j^{\prime}, j}= & \frac{1}{\mathcal{N}} \int \mathcal{D}[\phi] \mathcal{D}\left[\phi^{\prime}\right] \exp \left\{-\int d^{d} x\left(\begin{array}{c}
\phi \\
\phi^{\prime}
\end{array}\right)^{T} \cdot\left(\begin{array}{cc}
\frac{-\Delta+V^{(2)}}{2} & \frac{-\alpha^{(1)} \otimes \alpha^{(1) *}}{2} \\
\frac{-\alpha^{(1) *} \otimes \alpha^{(1)}}{2} & \frac{-\Delta+V^{(2) *}}{2}
\end{array}\right) \cdot\left(\begin{array}{c}
\phi \\
\phi^{\prime}
\end{array}\right)\right. \\
& \left.+\left(\begin{array}{c}
V^{(1)}-\alpha^{(1)}\left(j+\alpha^{(0) *}\right) \\
V^{(1)}-\alpha^{(1) *}\left(j^{\prime}+\alpha^{(0)}\right)
\end{array}\right)^{T} \cdot\left(\begin{array}{c}
\phi \\
\phi^{\prime}
\end{array}\right)-j \alpha^{(0)}-j^{\prime} \alpha^{(0) *}-\alpha^{(0)} \alpha^{(0) *}+V^{(0)}+V^{(0) *}\right\} .
\end{aligned}
$$

Carrying the Gaussian integration, we then obtain

$$
\mathcal{Z}_{j, j^{\prime}}=\frac{1}{\tilde{\mathcal{N}}} \exp \left\{\int d^{d} x d^{d} y \frac{1}{2} \Lambda\left(j, j^{\prime}\right)^{T}(x) \cdot K(x, y) \cdot \Lambda\left(j, j^{\prime}\right)(y)+\delta(x-y)\left(j \alpha^{(0)}+j^{\prime} \alpha^{(0) *}\right)(y)\right\},
$$

where

$$
\Lambda\left(j, j^{\prime}\right)=\left(\begin{array}{c}
V^{(1)}-\alpha^{(1)}\left(j+\alpha^{(0) *}\right) \\
V^{(1)}-\alpha^{(1) *}\left(j^{\prime}+\alpha^{(0)}\right)
\end{array}\right) \quad \text { and } \quad\left(\begin{array}{cc}
-\triangle+V^{(2)} & -\alpha^{(1)} \otimes \alpha^{(1) *} \\
-\alpha^{(1) *} \otimes \alpha^{(1)} & -\triangle+V^{(2) *}
\end{array}\right) K(x, y)=\mathbb{1}_{2 D \times 2 D} \delta(x-y) .
$$

Because of translation invariance, $K(x, y)=K(x-y)$, which can be written in Fourier space:

$$
K(x-y)=\int d^{d} p e^{i p \cdot(x-y)} K(p) .
$$

Inserting this expression into Eq. (75) and integrating over the variable $u=(x-y)$ yields

$$
K(p)=\frac{1}{(2 \pi)^{d}}\left(\begin{array}{cc}
p^{2}+V^{(2)} & -\alpha^{(1)} \otimes \alpha^{(1) *} \\
-\alpha^{(1) *} \otimes \alpha^{(1)} & p^{2}+V^{(2) *}
\end{array}\right)^{-1},
$$

which is difficult to make more explicit but could be computed exactly for given $\alpha$ and $V$. To get an intuition of the behavior of the two point functions, we may instantiate this expression on a simple example where the bond-field dimension $D$ equals 1 and $\alpha^{(0)}=V^{(1)}=0$ for simplicity. In that case, we have 


$$
K(p)=\frac{1}{(2 \pi)^{d}} \frac{1}{\left(p^{2}+V^{(2)}\right)\left(p^{2}+V^{(2) *}\right)-\left|\alpha^{(1)}\right|^{4}}\left(\begin{array}{cc}
p^{2}+V^{(2) *} & \left|\alpha^{(1)}\right|^{2} \\
\left|\alpha^{(1)}\right|^{2} & p^{2}+V^{(2)}
\end{array}\right) .
$$

Using Eq. (9) gives the correlation function:

$$
\begin{aligned}
C(x-y):=\left\langle\psi^{\dagger}(x) \psi(y)\right\rangle & =\frac{\left|\alpha^{(1)}\right|^{2}}{2}\left[(1,0) K(x, y)(0,1)^{T}+(0,1) K(x, y)(1,0)^{T}\right] \\
& =\frac{1}{(2 \pi)^{d}} \int d^{d} p \frac{\left|\alpha^{(1)}\right|^{4} e^{i p \cdot(x-y)}}{\left(p^{2}+V^{(2)}\right)\left(p^{2}+V^{(2) *}\right)-\left|\alpha^{(1)}\right|^{4}} .
\end{aligned}
$$

Importantly here, the correlation function in momentum space $C(p) \propto p^{-4}$ when $p \rightarrow+\infty$. Hence, the integral is not UV divergent for $x=y$ so long as $d \leq 3$, in which case the particle density $\left\langle\psi^{\dagger}(x) \psi(x)\right\rangle$ is finite.

\section{B. Non-Gaussian states}

For a non-Gaussian CTNS, it is no longer possible to compute the correlation functions exactly, in general. Furthermore, the definitions we provided for the CTNSs in Eqs. (1) or (14) are generically divergent. Nonetheless, one can use approximations or numerical techniques coming from the quantum field theory and tensor network toolboxes.

\section{Regularization and renormalization}

In the general case, the ansatz we put forward suffers from the same UV divergences that plague quantum field theories. As in QFTs, these divergences are in a way inevitable: The gradient squared $(\nabla \phi)^{2}$ in the path integral insufficiently penalizes high momenta in $d \geq 1$ (note that, again, the $d=1$ case is trivial). On the other hand, the locality of the underlying tensor network forbids higher derivatives. Hence, as in QFTs, the divergences are tied to the very property (locality) that we require.

Given this state of affairs, there are essentially three options to deal with divergences, depending on what one needs the state for.

The first option is simply to regularize the state with a momentum cutoff $\Lambda$, either directly in the path integralwhich breaks locality and destroys the operator representation - or in the operator representation-which generically breaks Euclidean invariance. In both cases, the scale $\Lambda$ is reminiscent of the inverse lattice spacing of discrete tensor networks. The parameters appearing in the expansion of $V$ and $\alpha$ are then the equivalent of the bare parameters in QFT Lagrangians. As long as the state is used as a variational ansatz, e.g., to minimize the energy of an anyway regularized QFT Hamiltonian, this fact is unproblematic. Indeed, carrying an optimization on bare or renormalized parameters is equivalent, and the fact that some properties break above a cutoff momentum is anyhow imposed by the physical QFT being approximated. In this approach, there is no restriction on the powers of the auxiliary field appearing in $V$ and $\alpha$.

One may also be interested in the class of CTNSs for their properties and not necessarily to approximate the ground state of a given system. In that case, going beyond regularization and renormalizing the state with proper counterterms and renormalization conditions seems necessary to preserve the locality of the underlying tensor network. In the general case, this procedure is equivalent to renormalizing a relativistic open quantum field theory, a problem which has received interest recently [45]. At the level of dimensional analysis, this procedure restricts powers of the auxiliary field in $V$ and $\alpha$ to renormalizable interactions and, hence, to the relevant and marginally relevant powers obtained in Sec. IV C. In $d=3$, it restricts the parameters to a finite number of tensors appearing in the finite polynomial expansion of $V$ and $\alpha$. Allowing for more auxiliary fields is thus necessary to make the CTNS class arbitrarily large and expressive in $d=3$.

Finally, a natural regularization may be provided by restricting the class of quantum states in $d-1$ on which the transfer matrix $\mathbb{T}$ acts. As we see, for special cases of $\mathbb{T}$, one can indeed recover finite results in $d=2$. In that case, the state itself is implicitly defined by the approximate method used to contract it.

\section{Dimensional reduction}

We now discuss the last option. To compute physical correlation functions in the general case, one can exploit their operator expression (20) given by the exponential of a transfer matrix acting in a space of one dimension less. In the $d=2$ case, the theory one needs to solve is thus simply a one-dimensional QFT. The latter is solvable with CMPSs (i.e., CTNSs in one dimension less), which, as a bonus, 
have a built-in UV regulator [46] and bring the computation back to a zero-dimensional problem [47]. We outline the steps of such a computation on a simple example.

We consider a CTNS on a torus $(\tau, x), \tau \in[0, T]$, $x \in[0, L]$ (as in Fig. 2), which reads, in the operator representation,

$$
\begin{aligned}
|V, \alpha\rangle= & \operatorname{tr}\left[\mathcal { T } \operatorname { e x p } \left(-\int_{0}^{T} d \tau \int_{0}^{L} d x \mathcal{H}(x)\right.\right. \\
& \left.\left.-\alpha[\hat{\phi}(x)] \psi^{\dagger}(\tau, x)\right)\right]|0\rangle .
\end{aligned}
$$

Correlation functions for this state at a fixed $\tau$ take a particularly simple form, with the propagator $\exp (\tau \mathbb{\pi})$ appearing only once. For example, the two-point function reads

$$
\left\langle\psi^{\dagger}\left(\tau, x_{1}\right) \psi\left(\tau, x_{2}\right)\right\rangle=\operatorname{tr}\left[\mathbb{1} \otimes \hat{\alpha}^{*}\left(x_{1}\right) \cdot \hat{\alpha}\left(x_{2}\right) \otimes \mathbb{1} \cdot e^{T \mathbb{\mathbb { T }}}\right] .
$$

Such $N$-point functions at equal $\tau$ contain useful information about the state in the thermodynamic limit $T \rightarrow+\infty$, $L \rightarrow+\infty$. Indeed, because of Euclidean invariance, they give access to all correlation functions of aligned points and a fortiori to all possible two-point functions, which is sufficient to compute the expectation values of most homogeneous and isotropic quasilocal Hamiltonians.

To simplify the discussion, we now consider the special case of an Hermitian $\mathbb{T}$ (obtained, e.g., when all the coefficients of $V$ and $\alpha$ are real). In the $T \rightarrow+\infty$ limit, $\exp (T \mathbb{T})$ is dominated by the projector on the eigenvector $|s s\rangle$ of $\mathbb{T}$ with the largest eigenvalue. The correlation functions then simplify, e.g.,

$$
\left\langle\psi^{\dagger}\left(\tau, x_{1}\right) \psi\left(\tau, x_{2}\right)\right\rangle \propto\left\langle\mathrm{ss}\left|\mathbb{1} \otimes \hat{\alpha}^{*}\left(x_{1}\right) \cdot \hat{\alpha}\left(x_{2}\right) \otimes \mathbb{1}\right| \mathrm{ss}\right\rangle .
$$

The right-hand side is the correlation function for $(2 \mathrm{D}$ copies of) a one-dimensional bosonic field theory, which motivates the use of a CMPS. More precisely, we use a CMPS defined on two copies of the auxiliary quantum fields to approximate the dominant eigenvector $|\mathrm{ss}\rangle$. Assuming only $D=1$ auxiliary field, we can write

$$
\begin{aligned}
\left|Q, R_{1}, R_{2}\right\rangle= & \operatorname{tr}\left[\mathcal { P } _ { x } \operatorname { e x p } \left\{\int_{0}^{L} d x Q \otimes \mathbb{1}\right.\right. \\
& \left.\left.+R_{1} \otimes \psi_{1}^{\dagger}(x)+R_{2} \otimes \psi_{2}^{\dagger}(x)\right\}\right]|0\rangle,
\end{aligned}
$$

where $Q, R_{1}$, and $R_{2}$ are $(\chi \times \chi)$ matrices, $\psi_{1}^{\dagger}=\psi_{[d-1]}^{\dagger} \otimes \mathbb{1}$ and $\psi_{2}^{\dagger}=\mathbb{1} \otimes \psi_{[d-1]}^{\dagger}$ are the creation operators associated to each copy of the Fock space on which $\mathbb{T}$ acts, $|0\rangle$ is the Fock vacuum of these two copies, and the trace is taken over the matrices. This object is nothing but a translation-invariant
CMPS for two species of bosons. The dominant eigenvector can then be approximated by choosing

$$
Q, R_{1}, R_{2}=\underset{Q, R_{1}, R_{2}}{\operatorname{argmax}} \frac{\left\langle Q, R_{1}, R_{2}|\mathbb{\mathbb { T }}| Q, R_{1}, R_{2}\right\rangle}{\left\langle Q, R_{1}, R_{2} \mid Q, R_{1}, R_{2}\right\rangle} .
$$

The right-hand side of Eq. (85) can be computed explicitly as a function of $Q, R_{1}$, and $R_{2}$. Indeed, in the same way as we compute the normal ordered correlation functions for a CTNS in Eq. (20), one can compute the normal ordered correlation functions for a CMPS, replacing $-\mathcal{H}(x)$ by $Q$ and $\alpha(x)$ by $R_{1}, R_{2}$ [34], e.g., for $x \geq y$ :

$$
\left\langle\psi_{1}^{\dagger}(x) \psi_{1}(y)\right\rangle=\operatorname{tr}\left[e^{(L-x) \top}\left(\mathbb{1} \otimes R_{1}^{*}\right) e^{(x-y) \top}\left(R_{1} \otimes \mathbb{1}\right) e^{y \top}\right]
$$

with the (zero-dimensional) transfer matrix:

$$
\mathrm{T}=Q \otimes \mathbb{1}+\mathbb{1} \otimes Q^{*}+R_{1} \otimes R_{1}^{*}+R_{2} \otimes R_{2}^{*} .
$$

One then just has to express $\mathbb{T}$ as a function of $\psi_{1}$ and $\psi_{2}$ instead of the field and conjugate momenta, which requires a choice, e.g.,

$$
\begin{aligned}
& \hat{\phi}(x)=\Lambda_{0}^{-1 / 2} \frac{\psi_{[d-1]}(x)+\psi_{[d-1]}^{\dagger}(x)}{\sqrt{2}}, \\
& \hat{\pi}(x)=\Lambda_{0}^{1 / 2} \frac{\psi_{[d-1]}(x)-\psi_{[d-1]}^{\dagger}(x)}{\sqrt{2} i},
\end{aligned}
$$

for some $\Lambda_{0}$. Taking the expectation value of products of local operators on the CMPS yields divergent contributions. They can be removed, e.g., by normal ordering $\mathcal{H}$ and $\alpha$ [48] in the operator representation of Eq. (14) or by adding a counterterm in the Hamiltonian as in Ref. [49]. In the end, the expectation value to maximize can be written

$$
\frac{\left\langle Q, R_{1}, R_{2}|\mathbb{T}| Q, R_{1}, R_{2}\right\rangle}{\left\langle Q, R_{1}, R_{2} \mid Q, R_{1}, R_{2}\right\rangle}=\frac{\operatorname{tr}\left[M\left(Q, R_{1}, R_{2}\right) e^{L T}\right]}{\operatorname{tr}\left[e^{L T}\right]},
$$

where $M\left(Q, R_{1}, R_{2}\right)$ is some polynomial of $Q, R_{1}$, and $R_{2}$ explicitly calculable from $V$ and $\alpha$. This expression can be simplified in the thermodynamic limit and then be maximized, e.g., by gradient ascent [34]. In practice, for transfer matrices with relativistic $\mathcal{H}$ like the ones we consider, this maximization has to be carried over matrices $Q, R_{1}$, and $R_{2}$ with a fixed maximum norm (or with a soft penalization of large norms). This constraint is necessary to prevent the CMPS and its finite entanglement from capturing only the UV features of the stationary state [46]. For sufficiently large bond dimension $\chi$, and taking into account this subtlety, we expect to get a good estimate of the stationary state. Once $Q$, $R_{1}$, and $R_{2}$ are fixed this way, physical correlation functions can be computed analytically using Eq. (83). For example, if $\alpha$ is linear $\alpha(\phi) \propto \phi$, we get, for $x \geq y$, 


$$
\begin{aligned}
& \left\langle\psi^{\dagger}(\tau, x) \psi(\tau, y)\right\rangle \propto \operatorname{tr}\left[e^{(L-x+y) \top}\left(R_{2} \otimes \mathbb{1}+\mathbb{1} \otimes R_{2}^{*}\right)\right. \\
& \left.\quad \times e^{(x-y) \top}\left(R_{1} \otimes \mathbb{1}+\mathbb{1} \otimes R_{1}^{*}\right)\right] .
\end{aligned}
$$

More complicated cases could be treated in a similar way. Through two successive dimensional reductions, we can thus compute certain correlation functions of a CTNS in $d=2$ with an expression involving only matrices with a finite number of entries $(d=0)$. There is a priori no objection, in principle, to contracting a $d=3$ CTNS this way, but each additional dimensional reduction is done at the price of a variational optimization. For numerical purposes, the optimization of the CMPS is the crucial step. While current methods $[46,49-51]$ can be used, the prospect to use CMPSs to solve field theories in more than one spatial dimension provides a strong additional motivation to make them more efficient.

\section{Perturbation theory}

Given that it is possible to compute correlation functions for Gaussian CTNSs, it is natural to compute correlation functions for more general states by carrying a perturbative expansion around Gaussian states. One simply Dyson expands the non-Gaussian part of the exponential in the expression for the generating functional (13). It generically yields an expansion in terms of Feynman diagrams, similar to that of QFT.

For example, if $\alpha[\phi]$ is linear in $\phi$ with a correction $\propto \lambda_{k \ell} \phi_{k} \phi_{\ell}$, the expansion contains diagrams composed of vertices with three and four legs, corresponding to the $\alpha[\phi] \alpha^{*}\left[\phi^{\prime}\right]$ term in Eq. (13), connected by Gaussian propagators. As previously mentioned, unless cancellations between different auxiliary fields occur, loop diagrams are UV divergent and a regularization is needed. We leave the derivation of the general Feynman rules, including a renormalization scheme, to future work. Notice that, in this approach, it is not the state itself that is defined through a perturbative expansion, but rather the correlation functions computed with it.

\section{Others}

There are, of course, many other ways one could compute correlation functions. As we mentioned before, one could rediscretize the CTNS to go back to a tensor network description, truncate the bond dimension, and use existing algorithms to contract it. However, this approach would seem to partially defeat the purpose of introducing the continuum in the first place. An interesting avenue is to explore known approximations or tools of the quantum field theory (besides the perturbation theory) that would not be obvious in the discrete, like saddle point approximations, large $D$ limits, or functional renormalization. Finally, direct Monte Carlo sampling of the auxiliary field, although it yields oscillating terms harming convergence in the general case, is a last-resort option.

\section{GENERALIZATIONS}

\section{A. General metric and anisotropy}

The main difficulty to overcome in order to construct CTNSs lies in preserving local Euclidean symmetries. We now wish to relax this constraint by allowing a general metric and anisotropic terms in the functional integral definition (1). Namely, it is natural to consider the following generalization.

Definition 4 (general functional integral formulation).A CTNS of a bosonic quantum field on a smooth Riemannian manifold $\mathcal{M}$ with boundary $\partial \mathcal{M}$ and metric $g$ is a state $|V, B, \alpha\rangle$ parametrized by two functions $V$ and $\alpha$ : $\mathbb{R}^{D+d D} \rightarrow \mathbb{C}$ and a boundary functional $B: L^{2}(\partial M) \rightarrow \mathbb{C}$ defined by the functional integral on an auxiliary $D$-component field $\phi$ :

$$
\begin{aligned}
|V, B, \alpha\rangle= & \int \mathcal{D} \phi B\left(\left.\phi\right|_{\partial \mathcal{M}}\right) \exp \left\{-\int_{\mathcal{M}} d^{d} x \sqrt{g}\right. \\
& \left.\times\left(\frac{g^{\mu \nu} \partial_{\mu} \phi_{k} \partial_{\nu} \phi_{k}}{2}+V[\phi, \nabla \phi]-\alpha[\phi, \nabla \phi] \psi^{\dagger}\right)\right\}|0\rangle,
\end{aligned}
$$

where all functions depend explicitly on the position and summation on $k$ is assumed.

\section{B. Specialization: CMERA}

A natural specialization of the previous generalization consists in having an auxiliary field living on an hyperbolic manifold $\mathcal{M}$ coupled to a physical field restricted to the boundary $\partial \mathcal{M}$ (see Fig. 5). Namely, we have in mind a state of the form

$$
\begin{aligned}
|V, \alpha\rangle \sim & \int \mathcal{D} \phi \exp \left\{-\int_{\mathcal{M}} \sqrt{g}\left(\frac{g^{\mu \nu} \partial_{\mu} \phi_{k} \partial_{\nu} \phi_{k}}{2}+V[\phi]\right)\right\} \\
& \times \exp \left\{\oint_{\partial \mathcal{M}} \alpha[\phi] \psi^{\dagger}\right\}|0\rangle,
\end{aligned}
$$
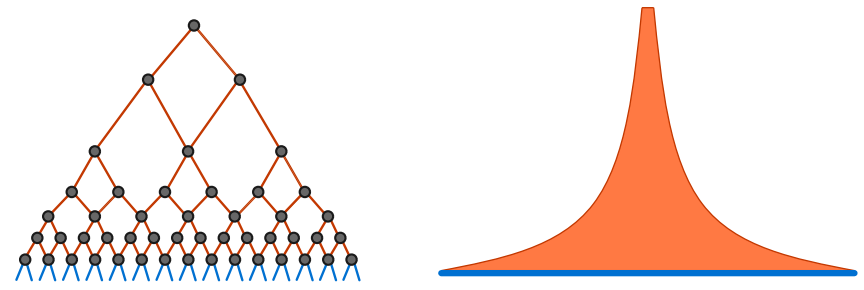

FIG. 5. Physical states on a boundary. In the discrete (left), the MERA is a (special case of a) tensor network state with a hierarchical structure, with physical indices only at the boundary. Tensor network states with such a structure can also be extended to the continuum with our CTNS ansatz, by restricting the physical field to the boundary and choosing an appropriate metric (here hyperbolic) for the bulk auxiliary fields. 
where the integral on the boundary may have to be taken as some appropriately rescaled limit of a bulk integral. Such states could provide a natural generalization of the MERA [32] in the continuum and for an arbitrary number of physical dimensions. They could provide a natural continuum versions of tensor network toy models of the AdS/CFT correspondence [29-31]. The form (93) is also reminiscent of field-theory toy models of the AdS/CFT correspondence [52], where scalar field theories on a fixed AdS background are related to conformal field theories on the boundary.

Note that this approach is different in spirit from that of the standard entanglement renormalization approach to quantum fields [53-57], constructed as a unitary transformation applied on a QFT ground state. In the proposal (93), there is a straightforward lattice discretization and a natural "bulk" description in terms of auxiliary fields. However, the isometry property, characteristic of the MERA, is less straightforward to implement.

\section{Fermions}

We define our ansatz for bosonic quantum fields, because functional integrals and field-coherent states are more natural in this context. To extend our proposal to fermions, one would have to introduce quite peculiar Grassmannian integrals with even kinetic and potential $V$ terms but a Grassmann odd term $\alpha$ in front of the creation operator $\psi^{\dagger}$. For fermions, it may be more convenient to start with an operator representation like that of Eq. (14), where Euclidean invariance is less natural, to subsequently derive the functional integral formulation.

\section{Conformal field theory}

We define CTNSs with the help of $D$ auxiliary free massless scalar fields of measure $d \mu$. A natural generalization would be to consider more general CFTs for the auxiliary space, in the spirit of what has been proposed in the context of matrix product states with infinite bond dimensions [41]. Admittedly, some nontrivial measures can already effectively be emulated by tuning the real part of the potential $V$ in Eq. (1). However, in the general case, it may be more convenient to use the CFT machinery directly, for example, on the wave function representation (7) and (17) or on correlation functions.

\section{DISCUSSION}

We put forward a new class of states for quantum fields that is obtained as a continuum limit of tensor network states and, thus, carries the same fundamental properties.

Although we show a number of interesting properties of our class of states, many interesting questions are so far open. Is it possible to find a quasilocal parent Hamiltonian for such states? Can the transfer matrix $\mathbb{T}$ used to compute correlation functions in the operator representation be put in canonical form? Are there important gauge transformations our discussion in Sec. IV B ignores? How do $V$ and $\alpha$ encode topological order and (local and global) gauge symmetries? Can this approach be combined with techniques developed on the lattice to study gauge theories with tensor networks [58-61]? Are there nontrivial nonGaussian CTNSs for which correlation functions can be computed exactly? Do (possibly regularized) CTNSs generically obey the area law like their discrete counterparts? Can CTNSs be used to construct interesting toy models of the AdS/CFT correspondence? To what extent does the bond-field dimension $D$ quantify entanglement for (possibly only some) classes of CTNSs?

Tackling these questions is an important goal for future work, to fully extend the success of tensor networks from the lattice to the continuum.

\section{ACKNOWLEDGMENTS}

We are grateful to Denis Bernard, Adrián Franco-Rubio, Giacomo Giudice, Anne Nielsen, German Sierra, Guifré Vidal, and Erez Zohar for helpful discussions. We thank two anonymous referees for valuable suggestions and comments. A. T. was supported by the Alexander von Humboldt Foundation and the Agence Nationale de la Recherche (ANR) Contract No. ANR-14-CE25-0003-01. The research of J. I. C. is partially supported by the ERC Advanced Grant QENOCOBA under the EU Horizon 2020 program (Grant Agreement No. 742102).

[1] J. I. Cirac and F. Verstraete, Renormalization and Tensor Product States in Spin Chains and Lattices, J. Phys. A 42, 504004 (2009).

[2] G. Evenbly and G. Vidal, Algorithms for Entanglement Renormalization: Boundaries, Impurities and Interfaces, J. Stat. Phys. 157, 931 (2014).

[3] A. Molnar, N. Schuch, F. Verstraete, and J. I. Cirac, Approximating Gibbs States of Local Hamiltonians Efficiently with Projected Entangled Pair States, Phys. Rev. B 91, 045138 (2015).

[4] M. B. Hastings, Solving Gapped Hamiltonians Locally, Phys. Rev. B 73, 085115 (2006).

[5] R. Orús, A Practical Introduction to Tensor Networks: Matrix Product States and Projected Entangled Pair States, Ann. Phys. (Amsterdam) 349, 117 (2014).

[6] R. Orús and G. Vidal, Infinite Time-Evolving Block Decimation Algorithm beyond Unitary Evolution, Phys. Rev. B 78, 155117 (2008).

[7] R. Orús, Exploring Corner Transfer Matrices and Corner Tensors for the Classical Simulation of Quantum Lattice Systems, Phys. Rev. B 85, 205117 (2012).

[8] M. Fannes, B. Nachtergaele, and R. F. Werner, Finitely Correlated States on Quantum Spin Chains, Commun. Math. Phys. 144, 443 (1992).

[9] S. R. White, Density Matrix Formulation for Quantum Renormalization Groups, Phys. Rev. Lett. 69, 2863 (1992). 
[10] S. R. White, Density-Matrix Algorithms for Quantum Renormalization Groups, Phys. Rev. B 48, 10345 (1993).

[11] U. Schollwöck, The Density-Matrix Renormalization Group, Rev. Mod. Phys. 77, 259 (2005).

[12] I. Niesen and P. Corboz, A Tensor Network Study of the Complete Ground State Phase Diagram of the Spin-1 Bilinear-Biquadratic Heisenberg Model on the Square Lattice, SciPost Phys. 3, 030 (2017).

[13] F. Verstraete and J. I. Cirac, Renormalization Algorithms for Quantum-Many Body Systems in Two and Higher Dimensions, arXiv:cond-mat/0407066.

[14] G. Vidal, Entanglement Renormalization, Phys. Rev. Lett. 99, 220405 (2007).

[15] S. Montangero, M. Rizzi, V. Giovannetti, and R. Fazio, Critical Exponents with a Multiscale Entanglement Renormalization Ansatz Channel, Phys. Rev. B 80, 113103 (2009).

[16] G. Evenbly and G. Vidal, Quantum Criticality with the Multi-Scale Entanglement Renormalization Ansatz, in Strongly Correlated Systems. Numerical Methods, edited by A. Avella and F. Mancini (Springer-Verlag, Berlin, 2013), Chap. 4, pp. 99-130.

[17] M. Srednicki, Entropy and Area, Phys. Rev. Lett. 71, 666 (1993).

[18] M. M. Wolf, F. Verstraete, M. B. Hastings, and J. I. Cirac, Area Laws in Quantum Systems: Mutual Information and Correlations, Phys. Rev. Lett. 100, 070502 (2008).

[19] J. Eisert, M. Cramer, and M. B. Plenio, Colloquium: Area Laws for the Entanglement Entropy, Rev. Mod. Phys. 82, 277 (2010).

[20] F. Pollmann, A. M. Turner, E. Berg, and M. Oshikawa, Entanglement Spectrum of a Topological Phase in One Dimension, Phys. Rev. B 81, 064439 (2010).

[21] X. Chen, Z.-C. Gu, and X.-G. Wen, Classification of Gapped Symmetric Phases in One-Dimensional Spin Systems, Phys. Rev. B 83, 035107 (2011).

[22] N. Schuch, D. Pérez-García, and I. Cirac, Classifying Quantum Phases Using Matrix Product States and Projected Entangled Pair States, Phys. Rev. B 84, 165139 (2011).

[23] X. Chen, Z.-C. Gu, Z.-X. Liu, and X.-G. Wen, Symmetry Protected Topological Orders and the Group Cohomology of Their Symmetry Group, Phys. Rev. B 87, 155114 (2013).

[24] N. Schuch, J. I. Cirac, and D. Pérez-García, PEPS as Ground States: Degeneracy and Topology, Ann. Phys. (Amsterdam) 325, 2153 (2010).

[25] N. Bultinck, M. Mariën, D. J. Williamson, M. B. Şahinoğlu, J. Haegeman, and F. Verstraete, Anyons and Matrix Product Operator Algebras, Ann. Phys. (Amsterdam) 378, 183 (2017).

[26] J. I. Cirac, D. Poilblanc, N. Schuch, and F. Verstraete, Entanglement Spectrum and Boundary Theories with Projected Entangled-Pair States, Phys. Rev. B 83, 245134 (2011).

[27] R. B. Laughlin, Anomalous Quantum Hall Effect: An Incompressible Quantum Fluid with Fractionally Charged Excitations, Phys. Rev. Lett. 50, 1395 (1983).

[28] X.-G. Wen, Edge Transport Properties of the Fractional Quantum Hall States and Weak-Impurity Scattering of a
One-Dimensional Charge-Density Wave, Phys. Rev. B 44, 5708 (1991).

[29] B. Swingle, Entanglement Renormalization and Holography, Phys. Rev. D 86, 065007 (2012).

[30] F. Pastawski, B. Yoshida, D. Harlow, and J. Preskill, Holographic Quantum Error-Correcting Codes: Toy Models for the Bulk/Boundary Correspondence, J. High Energy Phys. 06 (2015) 149.

[31] P. Hayden, S. Nezami, X.-L. Qi, N. Thomas, M. Walter, and Z. Yang, Holographic Duality from Random Tensor Networks, J. High Energy Phys. 11 (2016) 009.

[32] G. Vidal, Class of Quantum Many-Body States That Can Be Efficiently Simulated, Phys. Rev. Lett. 101, 110501 (2008).

[33] F. Verstraete and J. I. Cirac, Continuous Matrix Product States for Quantum Fields, Phys. Rev. Lett. 104, 190405 (2010).

[34] J. Haegeman, J. I. Cirac, T. J. Osborne, and F. Verstraete, Calculus of Continuous Matrix Product States, Phys. Rev. B 88, 085118 (2013).

[35] D. Jennings, C. Brockt, J. Haegeman, T. J. Osborne, and F. Verstraete, Continuum Tensor Network Field States, Path Integral Representations and Spatial Symmetries, New J. Phys. 17, 063039 (2015).

[36] P. Caputa, N. Kundu, M. Miyaji, T. Takayanagi, and K. Watanabe, Anti-de Sitter Space from Optimization of Path Integrals in Conformal Field Theories, Phys. Rev. Lett. 119, 071602 (2017).

[37] P. Caputa, N. Kundu, M. Miyaji, T. Takayanagi, and K. Watanabe, Liouville Action as Path-Integral Complexity: From Continuous Tensor Networks to ADS/CFT, J. High Energy Phys. 11 (2017) 097.

[38] M. E. Peskin and D. V. Schroeder, An Introduction to Quantum Field Theory (CRC Press, Boca Raton, FL, 1995).

[39] J. Zinn-Justin, Path Integrals in Quantum Mechanics (Oxford University, New York, 2010).

[40] G. Moore and N. Read, Nonabelions in the Fractional Quantum Hall Effect, Nucl. Phys. B360, 362 (1991).

[41] J. I. Cirac and G. Sierra, Infinite Matrix Product States, Conformal Field Theory, and the Haldane-Shastry Model, Phys. Rev. B 81, 104431 (2010).

[42] F. Verstraete, V. Murg, and J.I. Cirac, Matrix Product States, Projected Entangled Pair States, and Variational Renormalization Group Methods for Quantum Spin Systems, Adv. Phys. 57, 143 (2008).

[43] J. C. Bridgeman and C. T. Chubb, Hand-Waving and Interpretive Dance: An Introductory Course on Tensor Networks, J. Phys. A 50, 223001 (2017).

[44] F. Verstraete, J. I. Cirac, J. I. Latorre, E. Rico, and M. M. Wolf, Renormalization-Group Transformations on Quantum States, Phys. Rev. Lett. 94, 140601 (2005).

[45] A. Baidya, C. Jana, R. Loganayagam, and A. Rudra, Renormalization in Open Quantum Field Theory. Part I. Scalar Field Theory, J. High Energy Phys. 11 (2017) 204.

[46] J. Haegeman, J. I. Cirac, T. J. Osborne, H. Verschelde, and F. Verstraete, Applying the Variational Principle to $(1+1)$ Dimensional Quantum Field Theories, Phys. Rev. Lett. 105, 251601 (2010).

[47] T. J. Osborne, J. Eisert, and F. Verstraete, Holographic Quantum States, Phys. Rev. Lett. 105, 260401 (2010). 
[48] While normal ordering $\mathcal{H}$ just amounts to a change of normalization in the functional integral, normal ordering $\alpha$ requires explicit counterterms.

[49] V. Stojevic, J. Haegeman, I. P. McCulloch, L. Tagliacozzo, and F. Verstraete, Conformal Data from Finite Entanglement Scaling, Phys. Rev. B 91, 035120 (2015).

[50] D. Draxler, J. Haegeman, F. Verstraete, and M. Rizzi, Continuous Matrix Product States with Periodic Boundary Conditions and an Application to Atomtronics, Phys. Rev. B 95, 045145 (2017).

[51] M. Ganahl and G. Vidal, Continuous Matrix Product States for Nonrelativistic Quantum Fields: A Lattice Algorithm for Inhomogeneous Systems, Phys. Rev. B 98, 195105 (2018).

[52] J. Penedones, Tasi Lectures on ADS/CFT, in New Frontiers in Fields and Strings (World Scientific, Singapore, 2015), Chap. 2, pp. 75-136.

[53] J. Haegeman, T. J. Osborne, H. Verschelde, and F. Verstraete, Entanglement Renormalization for Quantum Fields in Real Space, Phys. Rev. Lett. 110, 100402 (2013).

[54] M. Nozaki, S. Ryu, and T. Takayanagi, Holographic Geometry of Entanglement Renormalization in Quantum Field Theories, J. High Energy Phys. 10 (2012) 193.
[55] A. Mollabashi, M. Naozaki, S. Ryu, and T. Takayanagi, Holographic Geometry of CMERA for Quantum Quenches and Finite Temperature, J. High Energy Phys. 03 (2014) 098.

[56] J. Cotler, M. R. Mohammadi Mozaffar, A. Mollabashi, and A. Naseh, Entanglement Renormalization for Weakly Interacting Fields, arXiv:1806.02835.

[57] J. Cotler, M. R. Mohammadi Mozaffar, A. Mollabashi, and A. Naseh, Renormalization Group Circuits for Weakly Interacting Continuum Field Theories, arXiv:1806.02831.

[58] E. Rico, T. Pichler, M. Dalmonte, P. Zoller, and S. Montangero, Tensor Networks for Lattice Gauge Theories and Atomic Quantum Simulation, Phys. Rev. Lett. 112, 201601 (2014).

[59] L. Tagliacozzo, A. Celi, and M. Lewenstein, Tensor Networks for Lattice Gauge Theories with Continuous Groups, Phys. Rev. X 4, 041024 (2014).

[60] J. Haegeman, K. Van Acoleyen, N. Schuch, J. I. Cirac, and F. Verstraete, Gauging Quantum States: From Global to Local Symmetries in Many-Body Systems, Phys. Rev. X 5, 011024 (2015).

[61] E. Zohar, M. Burrello, T. B. Wahl, and J. I. Cirac, Fermionic Projected Entangled Pair States and Local U(1) Gauge Theories, Ann. Phys. (Amsterdam) 363, 385 (2015). 